Original Contribution

\title{
MULTIDETECTOR COMPUTED TOMOGRAPHY APPROACH TO THE DIAGNOSIS OF A FOREIGN BODY
}

\author{
M. A. Mitev ${ }^{1 *}$, N. Trajkova ${ }^{2}$, D. Arabadzhiev ${ }^{3}$, S. Valkanov $^{4}$, N. Georgieva ${ }^{5}$, \\ E. Obretenov ${ }^{6}$ \\ ${ }^{1}$ Department of Diagnostic Imaging, University Hospital “St. Kirkovich”, Trakia University, \\ Stara Zagora, Bulgaria \\ ${ }^{2}$ Department of Diagnostic Imaging, University Hospital "St. George", Medical University, Plovdiv, \\ Bulgaria \\ ${ }^{3}$ Department of Pulmonology, University Hospital "St. Kirkovich", Trakia University, Stara Zagora, \\ Bulgaria \\ ${ }^{4}$ Department Surgery, Neurosurgery and Urology and Anesthesiology, Clinic of Neurosurgery, \\ Trakia University, Stara Zagora, Bulgaria \\ ${ }^{5}$ Department of Medical Physics, Biophysics, Roentgenology and Radiology, Trakia University, \\ Stara Zagora, Bulgaria \\ ${ }^{6}$ Department of Special Surgery / Thoracic Surgery, Vascular Surgery, Pediatric Surgery and \\ Orthopedics and Traumatology / Clinic of Thoracic Surgery, Trakia University, Stara Zagora, Bulgaria
}

\begin{abstract}
Introduction: The introduction of a foreign body in the respiratory tract is a serious global problem requiring urgent medical assistance. Purpose: The aim of the study is to assess the applicability of the Virtual bronchoscopy with MDCT for patients (adults and children) with suspected foreign body. Methods: To all 9 patients Virtual bronchoscopy and Fiberoptic bronchoscopy were carried out. The studies were carried out with an optimized protocol. In order to compare the results of FB and VB the sensitivity and precision indicators were determined. Results: When comparing the results of the study of patients with suspected foreign body, both methods established 4 positive results each (44.45\%). Conclusions: VB is particularly reliable and suitable for children and adults with poor general condition. The results of VB with MDCT could be used for early diagnosis of suspected foreign bodies.
\end{abstract}

Key words: foreign body, Multidetector computed tomography, Virtual bronchoscopy

\section{INTRODUCTION}

The introduction of a foreign body in the respiratory tract is a serious global problem requiring urgent medical assistance. Foreign bodies are common findings, especially under the age of three years, but the incidence increases with age due to sickness or deterioration of the swallowing reflex (1-5, etc). The Virtual bronchoscopy (VB) is able to assess bronchial stenoses and occlusions caused by both endolumenal pathology (tumor, mucous, foreign body) and external compression (anatomical structures, tumor, lymph nodes) (6, etc.). Low-dose Multidetector computed tomography (MDCT) and VB are

\footnotetext{
*Correspondence to: Mitko Mitev, Department of Diagnostic Imaging, University Hospital "St.

Kirkovich”, Trakia University - Stara Zagora, 6000 Mobile: +35988770 6079, E-mail:

mitev.mitko69@gmail.com
}

non-invasive methods, which undoubtedly can be used to study suspected foreign body. Because of the fact that the multi-planar reconstructions enable accurate special determination of the foreign body, as well as of the changes in surrounding structures, MDCT and VB have priority over FB (Fiberoptic bronchoscopy; 7 , etc.). The new generations of MDCT scanners can be used to evaluate the dynamic changes of the respiratory tract and to obtain information on the change of indirect characteristics in case of foreign bodies. The multi-planar 2- and 3-dimensional techniques to restore the volume, including external representation and VB complement the conventional axial CT images to display changes in the respiratory tract in case of foreign bodies. 


\section{PURPOSE}

This study aims to assess the applicability of the VB with MDCT for patients (adults and children) with suspected foreign body.

\section{MATERIAL AND METHODS}

Scientific studies of 9 patients (5 men and 4 women aged 16 - 78 years; Table 1) with suspected foreign body, divided by age into five groups (I group - up to 25 years; II group 26-35 years; III group - 36-55 years; IV group - 56-75 years; V group - over 75 years) were carried out.

Table 1. Sex and age of examined patients

\begin{tabular}{|l|l|l|l|l|l|l|l|l|l|}
\hline N0. & $\mathbf{1}$ & $\mathbf{2}$ & $\mathbf{3}$ & $\mathbf{4}$ & $\mathbf{5}$ & $\mathbf{6}$ & $\mathbf{7}$ & $\mathbf{8}$ & $\mathbf{9}$ \\
\hline Sex & M & M & M & M & M & F & F & F & F \\
\hline Age & 52 & 55 & 33 & 78 & 55 & 42 & 16 & 58 & 75 \\
\hline
\end{tabular}

Legend: $\mathbf{M}$ - Male; $\mathbf{F}$ - Female

To all patients Virtual bronchoscopy and Fiberoptic bronchoscopy were carried out. For FB a "Philips Essenca" apparatus was used, and for VB - "Olympus BF PE2" and "Fujinon EP-120T". For VB the study was conducted on a MDCT of the company "Philips Essenca" and 64 MDCT of the company "Siemens Definition AS". The further processing of the images was performed on a postprocessing workstation "Siemens Singovia VB10" capable of tracking and comparing the images in the axial, coronal and sagittal plane. The studies were performed with an optimized protocol. In order to compare the results of FB and VB the sensitivity and precision indicators were determined. In all cases of variation in the values of the compared criteria, rank and Average \pm SD (Standard Deviation) were defined ("Microsoft Exel/Windows ${ }^{\circledR}$ XP Home Edition").

\section{RESULTS}

Subjects of the presented study were 9 patients (5 men, 55\% and 4 women, $45 \%$ ) aged 16-78 years $(51.56 \pm 19.41)$. The largest proportion were patients from the III age group (4, $40 \%$; three men and one woman), followed by those of the IV age group (3,30\%; 1 man and 2 women). The patients from the I and II age groups were represented by one patient each (10\%, respectively a woman and a man) (Table 1-2).

Table 2. Number of patients on mechanism of occurrence, sex and age

\begin{tabular}{|c|c|c|c|c|c|c|c|c|c|c|}
\hline Age groups & \multicolumn{2}{|l|}{$\mathbf{I}$} & \multicolumn{2}{|l|}{ II } & \multicolumn{2}{|c|}{ III } & \multicolumn{2}{|c|}{ IV } & \multicolumn{2}{|l|}{$\mathbf{V}$} \\
\hline Sex & $\mathbf{M}$ & $\mathbf{F}$ & $\mathbf{M}$ & $\mathbf{F}$ & $\mathbf{M}$ & $\mathbf{F}$ & $\mathbf{M}$ & $\mathbf{F}$ & $\mathbf{M}$ & $\mathbf{F}$ \\
\hline Alimentaria foreign bodies & & & $1^{2}$ & & & & & $2^{3,4}$ & $1^{1}$ & \\
\hline Aspirated secretions & 1 & & & & 2 & 1 & 1 & & & \\
\hline
\end{tabular}

According to the mechanism of occurrence, patients with foreign bodies were divided into two groups (Table 2): the first group - four patients with alimentary foreign bodies (a ${\text { chicken } \text { bone }^{1} \text {; a chestnut }}^{2}$; a denture ${ }^{3}$; an olive $\left.^{4}\right)$. Second group - five patients composed of adults and children having aspirated secretions. In one case with alimentary foreign bodies the location of the foreign body was in the upper third of the esophagus, while in the other two cases - in the intermediate bronchus in the right and in the right lower lobe bronchus. When comparing the results of the study of patients with suspected foreign body, both methods established 4 positive results each. They relate to the four patients with alimentary foreign bodies. The other five patients were diagnosed as negative by $\mathrm{FB}$, but were determined as false-positive from the results obtained by the VB. Therefore, the sensitivity and precision of FB in diagnosing patients with suspected foreign body aspiration is $100 \%$ and of VB $45 \%$. The results obtained in this study from VB and MDCT reconstructions were compared with those of FB. The comparative examination was done according to the distribution of patients into two groups depending on the mechanism of occurrence of the foreign body.

The first group of patients (Figures 1-4) was with alimentary foreign bodies associated with eating disorders in adults. For one of the patients, according to the case history, after breaking a fragment of a denture and after coughing the aspiration of the fragment followed. On the MDCT made in the axial images and the implemented multi-planar 
reconstructions in the area of the right lower lobe bronchus heterodense substance was diagnosed with the presence of an irregular centrally located area with bone equivalent density. On the VB full obturation of the right lower lobe bronchus was visible. Distally the section could not be traced. On the FB of the right lower lobe bronchus numerous irregular and thick mucus secretions were described in which after aspiration an irregularly shaped fragment of dental prosthesis was seen, with presence of hyperemia of the environmental mucosa and many secretions in distal direction too. In the second case, an adult patient according to the case history, choked at mealtimes and aspirated food. Followed hearty cough that lasted for days. The patient went for a clinical examination two weeks after the incident due to worsening of breathing and continuous coughing. When Computed tomography (CT) study was made, in the right intermediate lobe bronchus heterodense substance was observed with density from 30 to 270 Hounsfield units (HE) with bone equivalent density in the middle, with irregular shape and hypoventilation of the middle part. In the subsequent VB obturation of the right intermediate lobe bronchus with irregular form was found. In subsequent virtual FB in the right intermediate lobe bronchus a big quantity of purulent secretions filling the bronchus was found. At aspiration of the secretions and penetration, distally a foreign body with bone density was registered, which was captured and removed. It was found that it was due to a whole chicken cervical vertebra. The mucous in the area was heavily altered, hyperemic with multiple purulent secretions, including distally. A third similar case of a foreign body was due to an olive stone, fallen into the right lower lobe bronchus. In a CT study obturations of the bronchus were found for the sixth segment, from heterodense substance with average density to $90 \mathrm{HE}$ and hypoventilation distally of the axillary incisions and multi-planar reconstructions made. In a FB the presence of an obstruction by a foreign body - an olive was found. The fourth of the cases of alimentary foreign bodies was due to a patient with age-related changes and impaired psychosomatic status. After a CT examination an oval foreign body was found in the upper third of the esophagus with a form resembling to a chestnut and a density of $110 \mathrm{HE}$. On the axial and multi-planar images the location of the foreign body in the upper third of the esophagus was visible, causing a slight dislocation of the back of the trachea in the area of the foreign body without causing breathing disturbances and hypoventilations distally. In the virtual esophagoscopy complete obliteration of the esophagus was found. The FB found a foreign body - a chestnut, corresponding completely in shape and location to the images made at the CT examination.

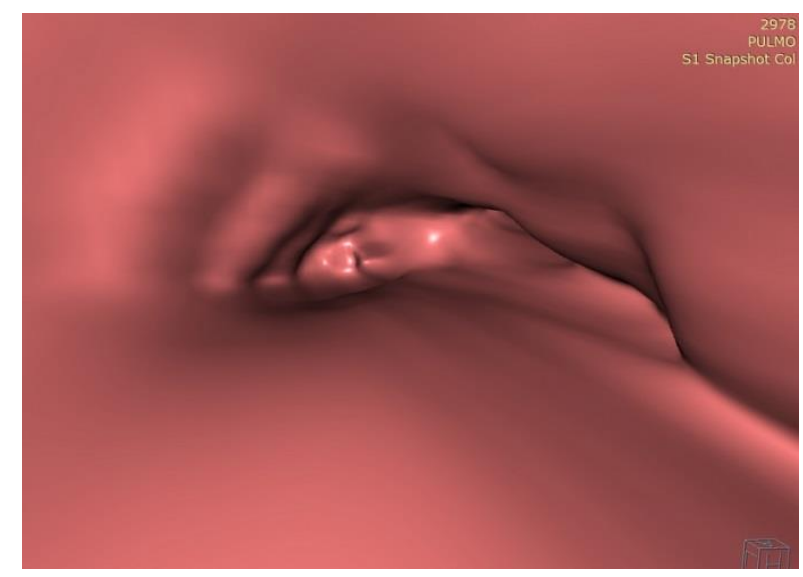

Figure 1. Obturation of intermediate bronchus with chicken vertebra by VB

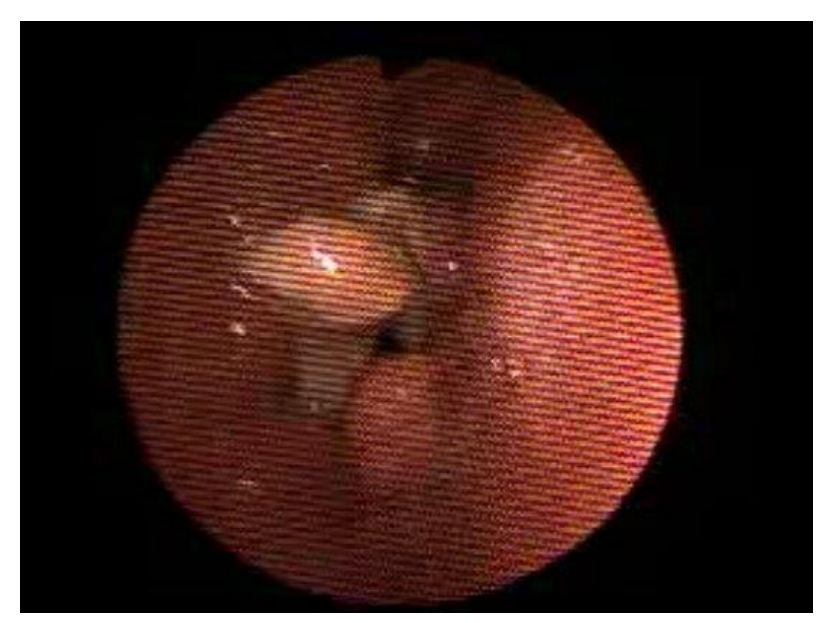

Figure 2. Obturation of intermediate bronchus with chicken vertebra by FB

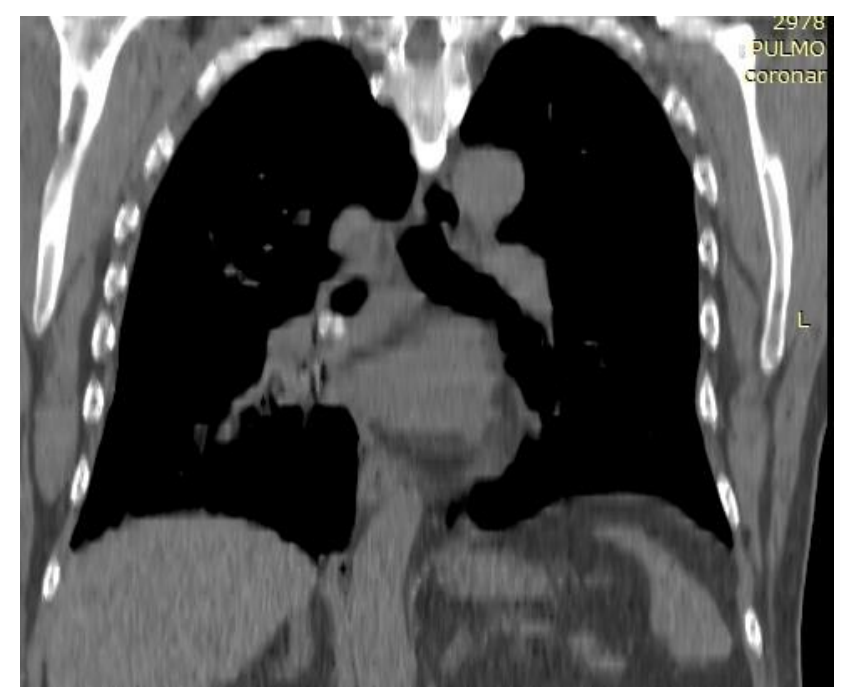

Figure 3. Obturation of intermediate bronchus with chicken vertebra - coronary projection 


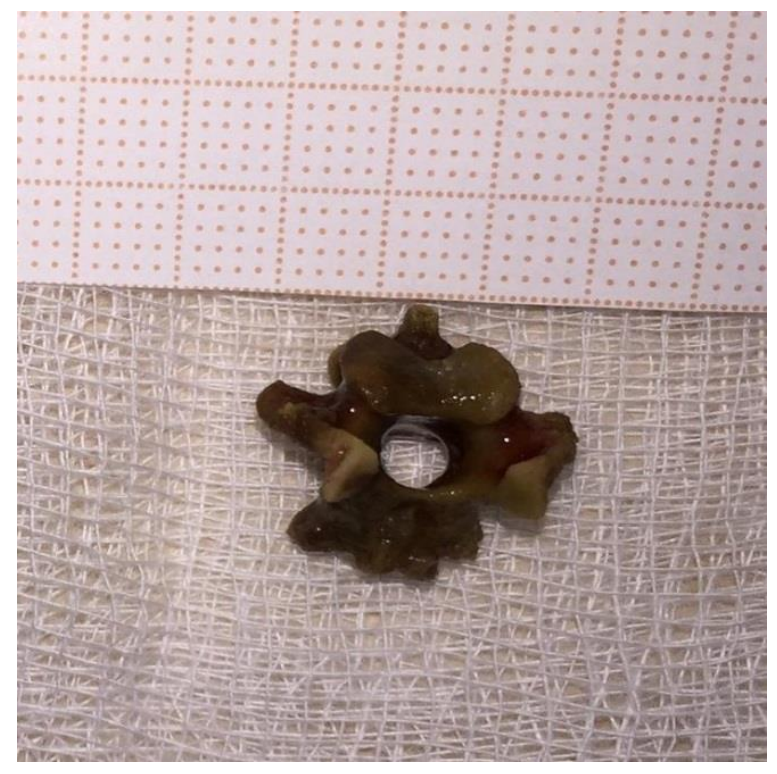

Figure 4. Chicken vertebra

The second group (Figures 5-7) was pediatric patients and adults with suspected aspiration of foreign bodies and infiltrative-inflammatory changes. At the CT studies in the area of the lung parenchyma in the right on the axial and multi-planar images the presence of a consolidation of the parenchyma with visible bronchograma, affecting one or several segments was found. In a VB, the trachea, the main and segmental bronchi were normal, with regular shape and size. In these cases obturation of the subsegmental bronchi in the right was visible. When making a real bronchoscopy, the trachea, and the main the segmental bronchi were normally passable. The presence of hyperemia of the mucosa was found, with thick mucus secretions distally, creating the possibility of a blockage of the small subsegmental bronchi and distal areas. After the aspiration thick mucus and purulent secretions were observed without evidence of foreign bodies from another source.

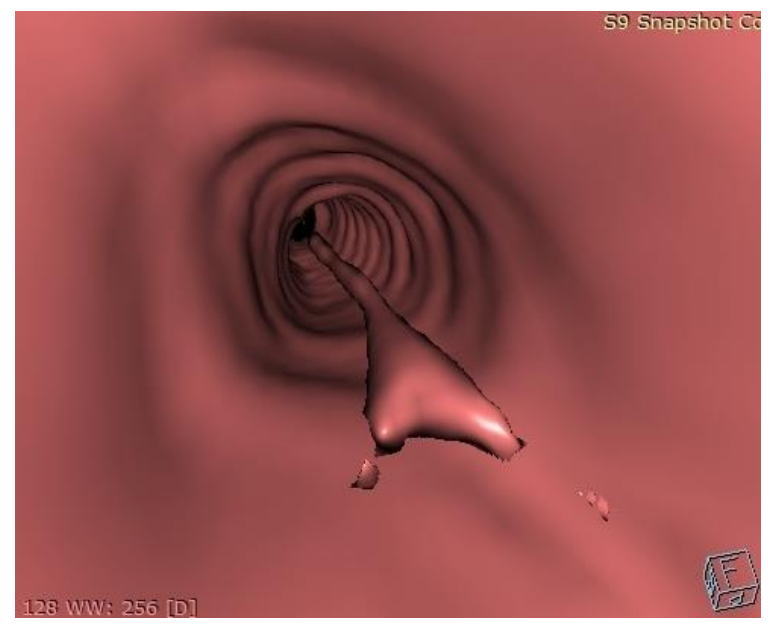

Figure 5. Mucus in the trachea by VB

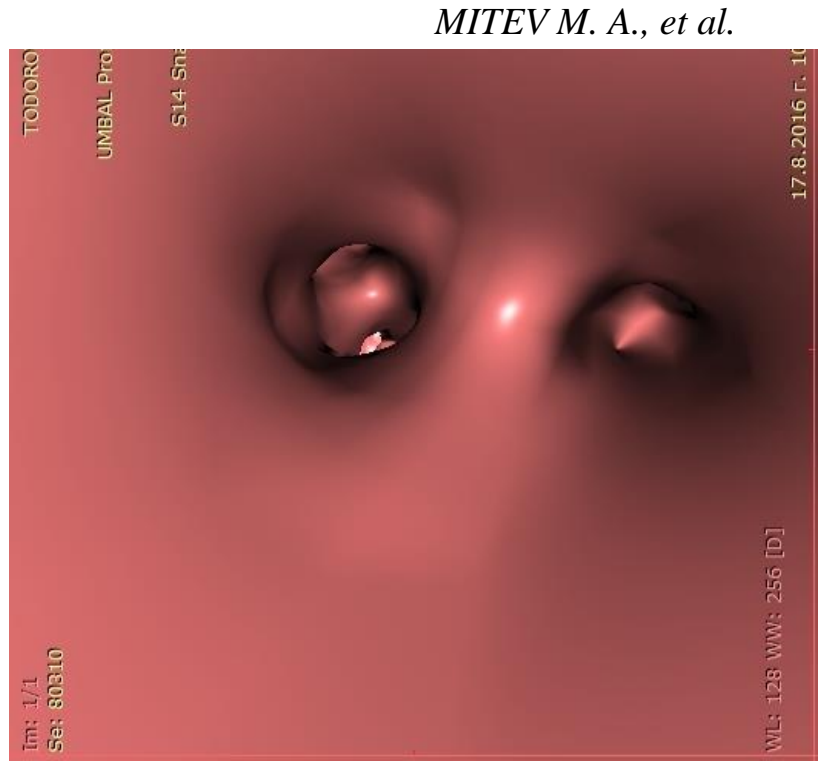

Figure 6. Obstruction of bronchus by secretions by VB

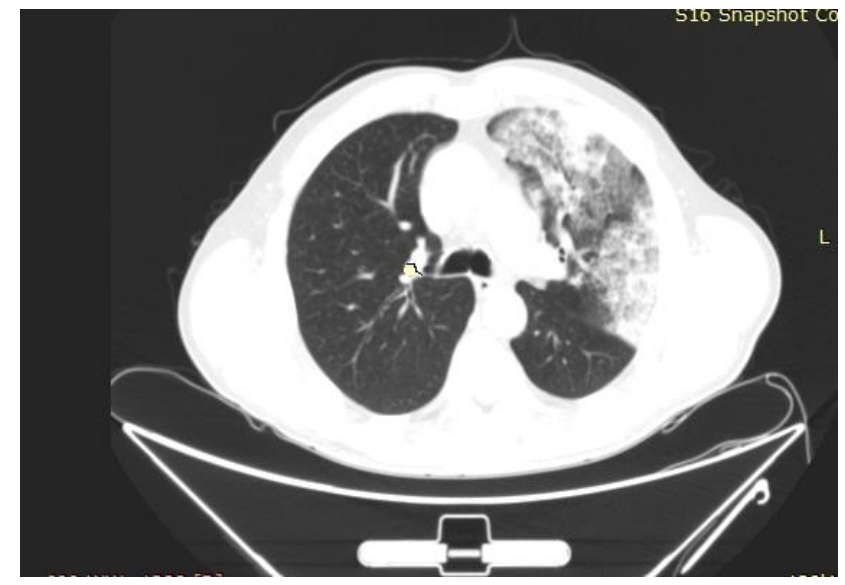

Figure 7. Hypoventilation parenchyma in clogging secretions - axial projection by MDCT

\section{DISCUSSION}

VB provides a detailed examination of the respiratory tract with minimal risk of infection or perforation and facilitates the preoperative planning of interventions. According to several studies, VB allows excellent results in terms of location, extent and shape of the narrowing of the respiratory tract. It is believed that VB will never replace FB ("the gold standard"), but it can assist and supplement it at shorting the operative time necessary to establish the exact location and size of the foreign body $(3,4,8,2$, etc). From the studies done it is clear that the benefits of the study with Multidetector 64 slices CT (MDCT 8) provide great opportunities for a comprehensive assessment of changes in the axial plane and for comparing the images to those in the multiplanar reconstructions and VB. The important role of the VB is determined as an opportunity for a preliminary assessment of the tracheobronchial tree as a source of valuable information about the findings and the 
subsequent real bronchoscopy. The study done shows the full compliance in the localization of foreign bodies; in particular in the studies submitted the changes affecting the main and segmental bronchi are located in the right. The shape of the foreign bodies cannot be determined accurately due to the presence of secondary inflammatory changes and secretions for which the method of VB with MDCT does not have the high sensitivity. For the low sensitivity in the VB in case of secretions contribute also the fact that in most cases study was done after a long period from the intake of the foreign bodies, 5-11 days, which led to the formation of secondary inflammatory changes and multiple mucus and purulent secretions. As a result of the accumulation of purulent secretions the distally located inflammations are visible as obstructive. As an advantage it is emphasized that the bronchi can be assessed distally and in adjacency. With the simultaneous interpreting of axial images and multi-planar reconstructions an idea is given of the endoluminal changes and the changes in the surrounding areas. The methodology is noninvasive and can be successfully used to diagnose foreign bodies in children and adults with poor general condition. Full compliance is seen of the results with those of the real bronchoscopy on the location of the finding. VB allows comprehensive assessment of the tracheobronchial tree with the changes around the lung parenchyma and the changes distally to the foreign bodies. The kind of foreign body and the changes in the mucosa cannot be determined only with the VB.

\section{CONCLUSION}

The accompanying axillary images and multiplanar reconstructions support the diagnosis and give an opportunity to interpret both the direct and indirect signs in case of foreign bodies. As a result of the axillary and multiplanar imaging the assessment is comprehensive, accurate and correct. CT VB is a non-invasive method that provides internal examination of the trachea and the main bronchi through a three-dimensional reconstruction. MDCT is essential to determine the exact location of the foreign body and for its timely removal. VB appears as a very useful method in the presence of a foreign body, but could not replace FB, it supports and supplements the results from FB. VB is particularly reliable and suitable for children and adults with poor general condition. The results of VB with MDCT could be used for early diagnosis of suspected foreign bodies.

\section{REFERENCES}

1. Kocaoglu M, Bulakbasi N, Soylu K, Demirbag S, Tayfun C \& Somuncu I. ThinSection Axial Multidetector Computed Tomography and Multiplanar Reformatted Imaging of Children with Suspected Foreign-Body Aspiration: Is Virtual Bronchoscopy Overemphasized? Acta Radiologica; 47(7):746-751, 2006.

2. Cevizci N, Dokucu Al, Baskin D, Karadağ CA, Sever N, Yalçin M, et al. Virtual bronchoscopy as a dynamic modality in the diagnosis and treatment of suspected forein body aspiration. Eur J Pediatr Surg; 18(6):398-401, 2008.

3. Kostadinov D. Foreign bodies in the trachea and bronchus. J MedInfo; 2011.

4. Boninska N, Borisova I. Imaging methods for detecting of foreign body in respiratory system. J Med forum; 3(4):71-74, 2014.

5. Jogi M, Surender K, Jadi L, Chavan S, Pratihiba P. Advantage of Computed Tomography (CT) Virtual Bronchoscopy in the Evaluation of Children with Suspected Foreign Body Airway. Int J Otolaryngol Head Neck Surg; 5:59-64, 2016.

6. Adali F, Uysal A, Bayramoglu S, Guner NT, Yilmaz G, Cimilli T. Virtual and fiberoptic bronchoscopy in patients with indication for tracheobronchial evaluation. Ann Thorac Med; 5(2):104-109, 2010.

7. Adaletli I, Kurugoglu S, Ulus S, Ozer H, Elicevik M, Kantarci F, et al. Utilization of low-dose multidetector $\mathrm{CT}$ and virtual bronchoscopy in children with suspected foreign body aspiration. Pediatr Radiol; 37(1):33-40, 2007.

8. Srivastava M, Tyagi S. Usefulness of virtual bronchoscopy in evaluation of suspected foreign body in tracheobronchial tree. Int J Otorhinolaryngol Head Neck Surg; 2(1):18-21, 2006. 